\title{
Effect of Huoxue Jiedu Jiangtang Formulation on Endoplasmic Reticulum Stress in Diabetic Atherosclerosis
}

\author{
Fu Xianzhao*, Huang Wenhua, Li Chunyan, Li Xingchan, Qiu Haixian, Liang Liudan, Cao Qiuxia, \\ Liang Liuyue
}

Clinic Medical College of Youjiang Medical National College, Baise, China

Email address:

1620100638@qq.com (Fu Xianzhao)

${ }^{*}$ Corresponding author

\section{To cite this article:}

Fu Xianzhao, Huang Wenhua, Li Chunyan, Li Xingchan, Qiu Haixian, Liang Liudan, Cao Qiuxia, Liang Liuyue. Effect of Huoxue Jiedu Jiangtang Formulation on Endoplasmic Reticulum Stress in Diabetic Atherosclerosis. American Journal of Clinical and Experimental Medicine. Vol. 6, No. 5, 2018, pp. 118-124. doi: 10.11648/j.ajcem.20180605.13

Received: October 31, 2018; Accepted: December 7, 2018; Published: December 27, 2018

\begin{abstract}
Objecive: To study the effects of Huoxue Jiedu Jiangtang formulation (HJJF) on endoplasmic reticulum stress (ERS) coupled inflammatory response From the molecular mechanism in diabetic vascular disease. Methods: Type 2 diabetes were established in male SD rats by feeding high lipid diet and injection of streptozotocin. after T2DM models established successfully, the rats were divided into model group, low-dose HJJF group $\left(\mathrm{HJJF}_{1}\right)$, high-dose $\mathrm{HJJF}$ group $\left(\mathrm{HJJF}_{2}\right)$, and Western medicine group (Gliquidone+Benazepril), and accepted corresponding drugs for 8 weeks respectively. Another normal group were used as control group, fed with normal diet, no drug intervention. The levels of fasting blood glucose (FBG), serum superoxide dismutase (SOD), glutathione peroxidase (GSH-Px), IL-6 and TNF- $\alpha$ were tested. The ERS signaling molecules glucose-regulated protein 78 (GRP78) and c-Jun N-terminal kinases (JNK) mRNA transcription level in thoracic aorta vessel were determined by reverse-transcription polymerase chain reaction (RT-PCR). Results: Compared with model group, After drugs intervention, all administered groups could significantly decrease FPG $(\mathrm{P}<0.05)$, elevate SOD and GSH-Px $(\mathrm{P}<0.05)$; all could reduce levels of TC, TG, LDL-C, IL-6 and TNF- $\alpha(P<0.05)$, and raise HDL-C $(P<0.05)$; all could depress the transcription levels of GRP78 and JNK mRNA $(\mathrm{P}<0.05)$. With increasing dose, the HJJF effect is more significant $(\mathrm{P}<0.05)$. Conclusion: HJJF could improve insulin resistance, correct lipid metabolism disorders, and enhance the antioxidative ability, inhibit the response of ERS coupled inflammatory, showing the multi-target treatment characteristics of Traditional Chinese Medicine.
\end{abstract}

Keywords: Diabetes, Atherosclerosis, Huoxue Jiedu Jiangtang Formulation, Inflammatory Reaction, Endoplasmic Reticulum Stress

\section{Introduction}

Diabetic atherosclerosis (AS) is a chronic inflammatoryrelated metabolic disease, and Endoplasmic reticulum stress (ERS) is a bridge between metabolic abnormalities and inflammatory reactions. In 2004, Ozcan U et al. [1] published an article in «Sciences», pointing out that ERS is a common pathway of multiple stressors and an important link in the occurrence and development of insulin resistance and complications in diabetes mellitus. In 2016 «Nature», Wang Met et al. [2] explained that ERS contributes to the aetiology of many human diseases. Therefore, ERS is expected to become a new target for the treatment of diabetes mellitus.
Although the pathogenesis of diabetic AS is complex, the pathological state of diabetes mellitus, such as high glucose, high fat, oxidative stress and inflammatory reaction, are related to ERS, which link the metabolic abnormalities and the development of diabetes mellitus, can cause apoptosis of macrophages and smooth muscle cells and participate in the occurrence and development of diabetic AS [3]. According to traditional Chinese medicine (TCM) theory, diabetes and coronary heart disease belong to the category of Xiao Ke and chest obstruction respectively, whose pathogenesis are deficiency of Qi and Yin, dry and heat holding in store, and with the passing of time, the fluid was burnt into phlegm, Yin deficiency leads to Yang insufficiency, blood coagulate into stasis, and finally, the accumulated phlegm and stasis turn to 
toxin. Therefore, deficiency of Qi and Yin, accumulation of blood stasis toxin are the main pathogenesis of diabetes and chest obstruction [4]. Previous clinical studies have found that Huoxue Jiedu Jiangtang formulation (HJJF), with the functions of Nourishing yin and replenishing qi, activating blood circulation and detoxicating, can significantly reduce pro-inflammatory factors, regulate anti-inflammatory/proinflammatory balance, effectively inhibit inflammation in non revascularization of diabetic acute coronary syndrome (critical coronary heart disease) patients, and improve their clinical curative effect [5]. In this study, animal models were used to investigate the molecular mechanism of ERS coupled with inflammatory response in the development of diabetic AS.

\section{Methods}

\subsection{Model Establishment}

120 male SD rats Weighing 220-250 g were fed (each cage hold four rats) at room temperature of $22-25^{\circ} \mathrm{C}$ and relative humidity of $40-50 \%$. After adapted feeding for 7 days, and fasted for 10 hours, the rats were intraperitonealy injected streptozotoein (STZ) (Beijing Hua Yang Biotechnology Co., Ltd.) once $50 \mathrm{mg} / \mathrm{kg}$. 72 hours later, blood glucose were tested with blood glucose test strips (Wuhan Boster Biological Technology, LTD). Animals with $\mathrm{FBG} \geq 16.7 \mathrm{mmol} / \mathrm{L}$ two consecutive times were used for diabetes models. Then the rats were fed with high fat diet (contain common feed $78.2 \%$, lard $10 \%$, cholesterol $1.5 \%$, porcine bile salt $0.3 \%$, custard powder $10 \%$ ) provided by Jiangsu synergetic Biological Engineering Co., Ltd) for 3 months. Daily food and water intake were recorded. Another 25 normal rats group were given intraperitoneal injection of equal amount of normal saline and common feed. Above rats were provided from experimental animal center of Guangxi Medical University, certificate number SCXK (GUI) 2014-002.

\subsection{Grouping and Administration of Rats}

Total of 102 rats were successfully modeled, which subsequently were randomly allocated into model group 25, HJJF low dose group $\left(\mathrm{HJJF}_{1}\right)$ 26, HJJF high dose group $\left(\mathrm{HJJF}_{2}\right) 25$, western medicine (Gliquidone+benazepril) group 26. In addition, 25 homology rats were taken as normal group, accepted intraperitoneal injection of normal saline, fed with ordinary feed. HJJF (Ginseng, Astragalus membranaceus, Ophiopogon japonicus, Cornus officinalis, Rehmannia glutinosa, Rhubarb, turtle shell, peach kernel, danpi, Coptis chinensis, Salvia miltiorrhiza, yam, Schisandra chinensis, suppled by Affiliated Hospital of Youjiang National Medical College) were made of extracts, $1 \mathrm{~g}$ of which equivalent to $10 \mathrm{~g}$ raw herb. HJJF1 Group were treated with $1 \mathrm{~g} \cdot \mathrm{kg}^{-1} \cdot \mathrm{d}^{-1}$ HJJF Suspensions, while the HJJF2 group were administered by $1.5 \mathrm{~kg}^{-1} \cdot \mathrm{d}^{-1}$ of which; the western medicine group were fed with benazepril (Beijing Novartis Pharma Ltd) $2.50 \mathrm{mg} / \mathrm{kg}$ and Gliquidone (Beijing Wan Hui Shuanghe Pharmaceutical Co Ltd) $7.50 \mathrm{mg} / \mathrm{kg}$, which were confected equal volume solution.
ALL rats were administered for 2 months and fed with high-fat diet. The model group was fed with high-fat diet only, while the normal group was fed with normal diet. The experimental operations were carried out in super-clean worktable.

\subsection{Experimental Sampling}

After 60 days of administration, fasting for 12 hours (drinking water frely) and FBG testing, rats in each group were anesthetized by intraperitoneal injection of $8 \%$ sodium pentobarbital at a dose of $2 \mathrm{~mL} / \mathrm{kg}$. Then blood samples $7-10 \mathrm{~mL}$ were collected by laparotomy and jugular vein puncture. Sera was separated by $3000 \mathrm{r} / \mathrm{min} 10 \mathrm{~min}$ centrifugation, and kept in $-20^{\circ} \mathrm{C}$ refrigerator for index detection. The aorta was peeled off, washed with saline, and stored at $-80^{\circ} \mathrm{C}$ for RT-PCR experiment.

\subsection{Index Determination}

\subsubsection{Detection of Serum Lipids, SOD, GSH-Px, IL-6 and TNF-a}

Total cholesterol (TC), triglyceride (TG), low density lipoprotein cholesterol (LDL-C) and high density lipoprotein cholesterol (HDL-C) were determined by automatic biochemical instrument. SOD content was tested by xanthine oxidase method, interleukin-6 (IL-6), tumor necrosis factor-alpha (TNF-a) and GSH-Px levels were checked by enzyme-linked immunosorbent assay (ELISA). The kits were purchased from Wuhan Doctor De Bioengineering Co., Ltd。 The specific experimental procedures were carried out according to the instructions

\subsubsection{Useing Real-Time PCR Method to Detect the Transcription of GRP78 and JNK $m$ RNA in Aorta}

Taking out the aortas from $-80 \mathrm{C}$ refrigerator, grinding in a mortar, extracting the total RNA, conducting electrophoresis in agarose gel, and checking its integrity, turn it into cDNA (at $42^{\circ} \mathrm{C} 50 \mathrm{~min}$, then $95^{\circ} \mathrm{C} 5 \mathrm{~min}$ ) by reverse transcription. The obtained cDNA was used as template to amplify the target gene by PCR (according to the instructions of Real-time PCR kit). The reference and target gene primers were designed and synthesized by Shanghai Genechem Co., LTD. The sequence of primers are as follows: GAPDH upstream 5'-TTCAACGGCACAGTCA AGG-3', downstream 5'-CTCAGCACCAGCATCACC-3', amplified product $114 \mathrm{bp}$; GRP78 upstream 5'-CTTGGTATTGAA ACTGTGGG-3', downstream 5'-TGTTACGGTGGGCTGATTAT-3',Amplified product $117 \mathrm{bp}$; c-JNK upstream 5'-GGATTTGGAGGAG CGAACTAA-3', downstream 5'-ACTGCTGTCTGTATCCG AGGC-3', amplified product $163 \mathrm{bp}$. The process of amplification of obtained cDNA is as follows: $96^{\circ} \mathrm{C} 4 \mathrm{~min}$, one cycle, subsequently $94^{\circ} \mathrm{C} 30$ second, $58^{\circ} \mathrm{C} 30$ second, $72^{\circ} \mathrm{C}$ 30 second, 40 cycle. The number of cycles when the set threshold reached (CT) were recorded, and the specificity of primers was detected by dissolution curve. With $2^{-\Delta \Delta} \mathrm{Ct}$ representing the expression of target gene in experimental group, $\Delta \Delta \mathrm{Ct}=$ Experience group $\left(\mathrm{Ct}_{\text {target gene }}-\mathrm{Ct}_{\mathrm{GAPDH}}\right)$-control group $\left(\mathrm{Ct}_{\text {target gene }}-\mathrm{Ct}_{\mathrm{GAPDH}}\right)$. 


\subsection{Statistical Analysis}

All data were expressed by mean \pm standard deviation $(\bar{X} \pm \mathrm{S})$ and SPSS 19.0 statistical software was used to process the data. One-way ANOVA was used to analyze the differences between groups. Homageneity of variance test was put into application to test the homogeneity of variance. If the variances were homogeneous, LSD was applied to calculate the variances, if not, Tambanes $\mathrm{T} 2$ is put to use. $\mathrm{P}<0.05$ was considered to indicate the statistically significant difference.

\section{Result}

\subsection{Changes of Experimental Process in Rats}

After successful modeled, rats urinated and drank more, with weight loss and withered hair, acting slow and sluggish. But the intragastric administration was successful and no rat died.

\subsection{Effects of HJJF on Activities of FBG, SOD, GSH-Px, TNF- $a$ and $I L-6$ in Rats}

The results showed that compared with the model group, in drug treated groups, FBG decreased significantly $(\mathrm{P}<$ $0.05)$, antioxidant enzymes SOD and GSH-Px increased ( $\mathrm{P}<$ $0.05)$, inflammatory factors TNF-a and IL-6 declined ( $\mathrm{P}<$ $0.05)$; HJF group had better curative effect than western medicine group, and the effect was more significant with the increasing HJF dose $(\mathrm{P}<0.05)$, but there was no significant difference between HJF groups $(\mathrm{P}>0.05)$. The results are shown in Table 1

Table 1. Effects of HJJF on FBG, SOD, GSH-Px, TNF- $a$ and IL-6 in rats $(\bar{X} \pm S)$.

\begin{tabular}{|c|c|c|c|c|c|c|}
\hline Group & $\mathbf{n}$ & FBG $(\mathrm{mmol} / \mathrm{L})$ & $\operatorname{SOD}(u / \mathbf{m L})$ & GSH-Px (u/mL) & TNF- $\alpha(n g / L)$ & IL-6 (ng/L) \\
\hline normal group & 25 & $5.19 \pm 0.71$ & $391.7 \pm 63.2$ & $3722.3 \pm 489.4$ & $51.64 \pm 2.86$ & $301.21 \pm 78.87$ \\
\hline model group & 25 & $16.71 \pm 1.63^{\mathrm{a}}$ & $265.2 \pm 49.7^{\mathrm{a}}$ & $4301.7 \pm 639.7^{\mathrm{a}}$ & $417.68 \pm 63.72^{\mathrm{a}}$ & $3151.58 \pm 857.21^{\mathrm{a}}$ \\
\hline $\mathrm{HJJF}_{1}$ group & 26 & $10.21 \pm 1.34^{\mathrm{ab}}$ & $328.0 \pm 51.9^{\mathrm{ab}}$ & $4548.3 \pm 489.6^{\mathrm{ab}}$ & $138.75 \pm 51.12^{\mathrm{ab}}$ & $912.32 \pm 231.32^{\mathrm{ab}}$ \\
\hline $\mathrm{HJJF}_{2}$ group & 25 & $9.77 \pm 1.55^{\mathrm{abc}}$ & $358.6 \pm 81.7^{\mathrm{abc}}$ & $4811.2 \pm 588.6^{\mathrm{abc}}$ & $116.51 \pm 45.56^{\mathrm{abc}}$ & $865.45 \pm 126.21^{\mathrm{abc}}$ \\
\hline western medicine group & 26 & $10.79 \pm 1.48^{\mathrm{ab}}$ & $305.1 \pm 41.7^{\mathrm{ab}}$ & $4440.3 \pm 478.7^{\mathrm{ab}}$ & $141.54 \pm 50.27^{\mathrm{ab}}$ & $956.38 \pm 261.36^{\mathrm{ab}}$ \\
\hline
\end{tabular}

Notes: Compared with the normal group, ${ }^{a} \mathrm{P}<0.01$; Compared with the model group, ${ }^{b} \mathrm{P}<0.05$; Compared with the western medicine group, ${ }^{\mathrm{c}} \mathrm{P}<0.05$

\subsection{Effect of HJJF on Blood Lipid in Rats}

Compared with the normal control group, the contents of TC, TG and LDL-C in the model group increased significantly $(\mathrm{P}<0.01)$, and HDL-C decreased significantly $(\mathrm{P}<0.01)$; compared with the model group, after administration, the contents of TC, TG and LDL-C decreased, and the contents of HDL-C increased in all drug treated groups $(\mathrm{P}<0.05)$. The effect of HJF group was more significant with the increase of dosage, and the difference was statistically significant compared with the model group. There was no significant difference between HJJF groups $(\mathrm{P}>0.05)$. The results are shown in Table 2

Table 2. Effects of HJJF on TC, TG, LDL-C and HDL-C ( $\bar{X} \pm S)$.

\begin{tabular}{llllll}
\hline Group & n & TC $(\mathbf{m m o l} / \mathbf{L})$ & TG $(\mathbf{m m o l} / \mathbf{L})$ & HDL-C $(\mathbf{m m o l} / \mathbf{L})$ & LDL-C $(\mathbf{m m o l} / \mathbf{L})$ \\
\hline normal group & 25 & $1.82 \pm 0.11$ & $0.91 \pm 0.12$ & $0.93 \pm 0.07$ & $0.91 \pm 0.52$ \\
model group & 25 & $5.38 \pm 0.41^{\mathrm{a}}$ & $2.31 \pm 0.24^{\mathrm{a}}$ & $0.52 \pm 0.06^{\mathrm{a}}$ & $2.11 \pm 0.75^{\mathrm{a}}$ \\
$\mathrm{HJJF}_{1}$ group & 26 & $2.92 \pm 0.31^{\mathrm{ab}}$ & $1.81 \pm 0.31^{\mathrm{ab}}$ & $0.78 \pm 0.07^{\mathrm{ab}}$ & $1.73 \pm 0.23^{\mathrm{ab}}$ \\
$\mathrm{HJJF}_{2}$ group & 25 & $2.81 \pm 0.30^{\mathrm{abc}}$ & $1.71 \pm 0.22^{\mathrm{abc}}$ & $0.81 \pm 0.09^{\mathrm{abc}}$ & $1.61 \pm 0.21^{\mathrm{abc}}$ \\
western medicine group & 26 & $2.96 \pm 0.33^{\mathrm{ab}}$ & $1.88 \pm 0.21^{\mathrm{ab}}$ & $0.75 \pm 0.08^{\mathrm{ab}}$ & $1.77 \pm 0.15^{\mathrm{ab}}$ \\
\hline
\end{tabular}

Notes: Compared with the normal group, ${ }^{\text {a }} \mathrm{P}<0.01$; Compared with the model group, ${ }^{\mathrm{b}} \mathrm{P}<0.05$; Compared with the western medicine group, ${ }^{\mathrm{c}} \mathrm{P}<0.05$

\subsection{Effects of HJJF on GRP78 and JNK $m R N A$ Transcription Levels in Rat Aorta}

According to the result of Table 3, compared with the normal control group, the expression of GRP78 and JNK in the model groups were significantly higher $(\mathrm{P}<0.01)$; after administration, the transcription of GRP78 and JNK in each group was lower than that in the model group $(\mathrm{P}<0.05)$; compared with the western medicine group, the effect of HJJF2 group was more significant $(\mathrm{P}<0.05)$; the effect of HJJF was better with the increase of dosage, but among the HJJF groups. The difference was not statistically significant $(\mathrm{P}>0.05)$. The results are shown in Table 3, Figure 1.

Table 3. Relative transcriptional level of GRP78 and JNK $m R N A$ in rats aorta in each group $\left(2^{-\Delta \Delta^{C t}}, \bar{X}_{ \pm S}\right)$.

\begin{tabular}{llll}
\hline Group & n & GRP78 & JNK \\
\hline normal group & 25 & $1.05 \pm 0.21$ & $1.07 \pm 0.26$ \\
model group & 25 & $7.12 \pm 0.38^{\mathrm{a}}$ & $7.63 \pm 0.24^{\mathrm{a}}$ \\
$\mathrm{HJJF}_{1}$ group & 26 & $4.21 \pm 0.87^{\mathrm{ab}}$ & $4.82 \pm 0.91^{\mathrm{ab}}$ \\
$\mathrm{HJJF}_{2}$ group & 25 & $3.15 \pm 0.37^{\mathrm{abc}}$ & $3.22 \pm 0.32^{\mathrm{abc}}$ \\
western medicine group $^{\mathrm{abc}}$ & $4.36 \pm 0.41^{\mathrm{ab}}$ & $4.94 \pm 0.41^{\mathrm{ab}}$ \\
\hline
\end{tabular}

Notes: Compared with the normal group, ${ }^{a} \mathrm{P}<0.01$; Compared with the model group, ${ }^{b} \mathrm{P}<0.05$; Compared with the western medicine group, ${ }^{\mathrm{c}} \mathrm{P}<0.05$ 


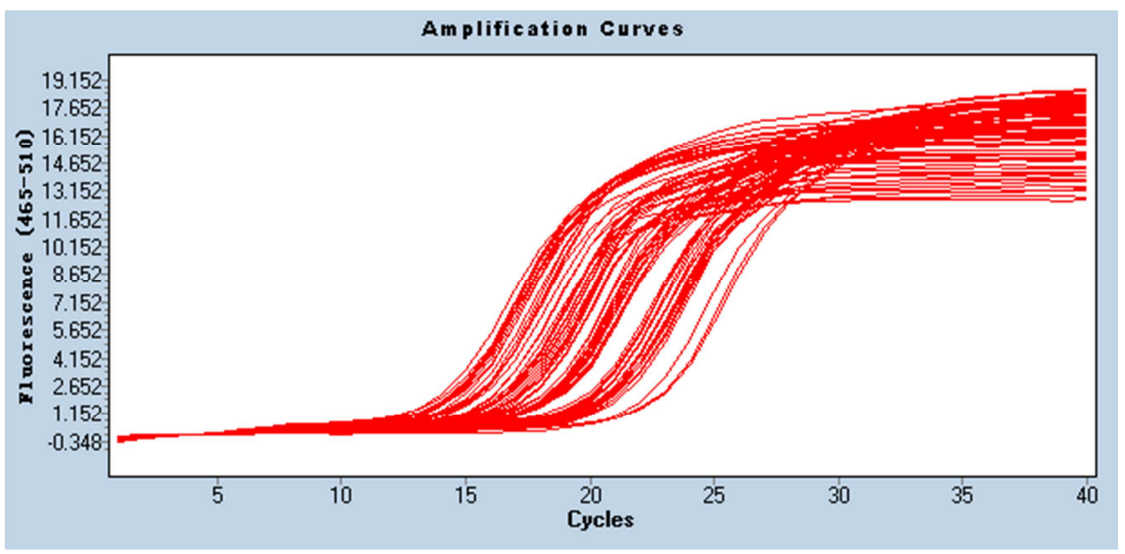

GRP78 amplification curve

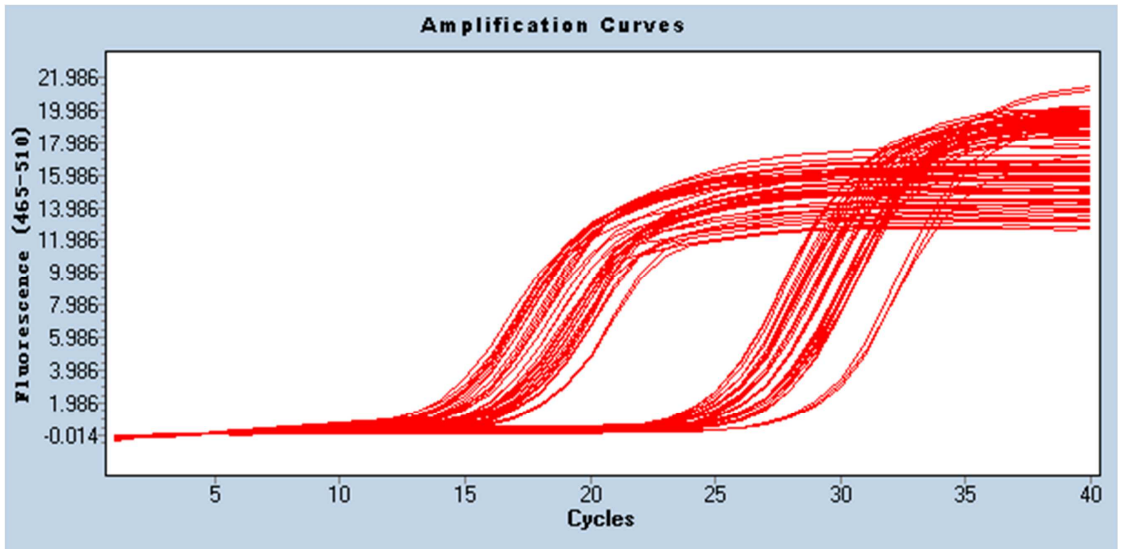

JNK amplification curve

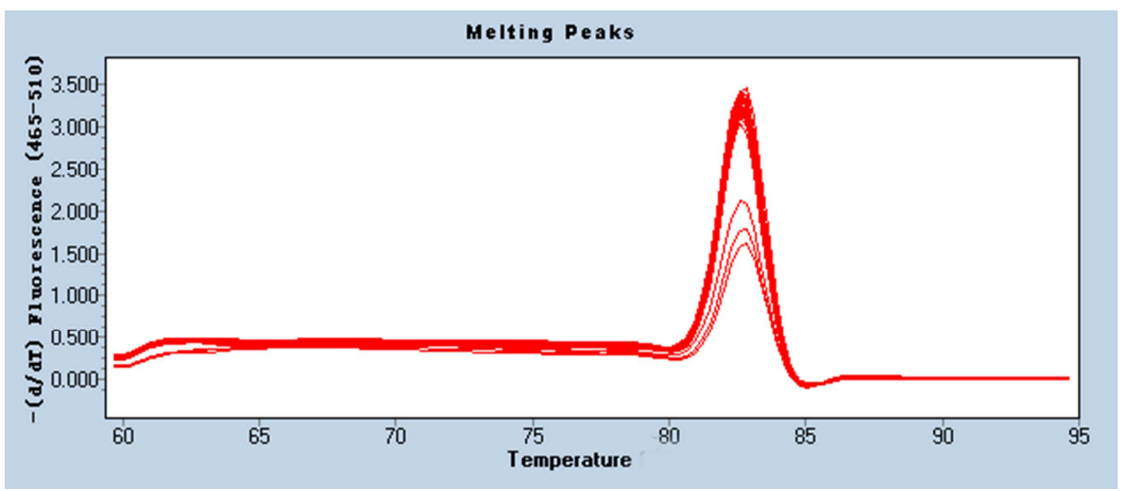

GRP78 dissolving curve

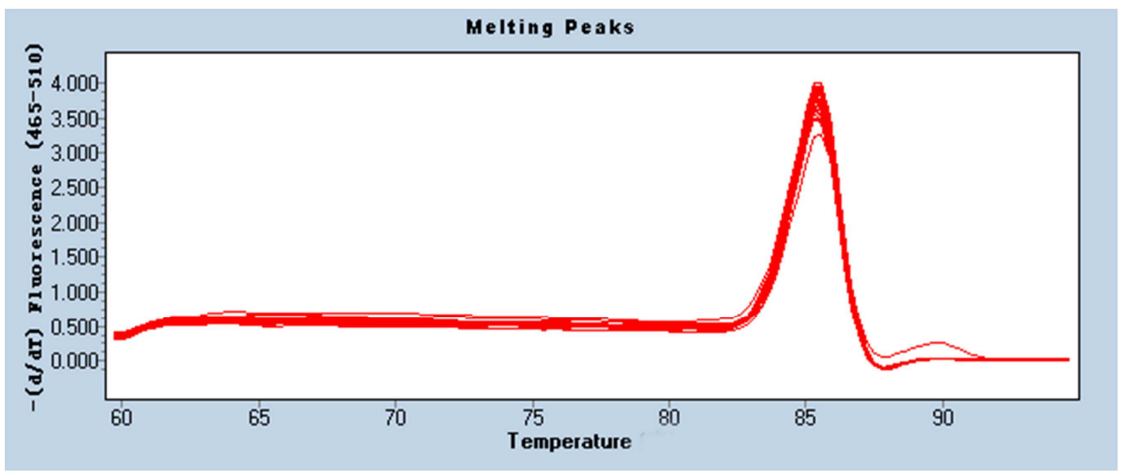

JNK dissolving curve

Figure 1. Amplification and dissolution curves of GRP78 and JNK mRNA in aorta. 


\section{Discussion}

Endoplasmic reticulum (ER) is an organelle for protein and steroid synthesis. When ER homeostasis imbalance leads to the accumulation of unfolded protein and disordered lipid synthesis, it will trigger a series of intracellular stress responses, called ERS [6]. Glucose regulated protein 78 (GRP78) is the feeling molecular for ER homeostasis. When ER is in steady state, GRP78 combined with the domain of three transmembrane proteins: inositol demand enzyme 1 (IRE-1), activating transcription factor 6 (ATF6) and protein kinase $\mathrm{R}$ like endoplasmic reticulum kinase (PERK), which exposed to the ER cavity. When severe ERS sustained, GRP78 will dissociate with PERK, ATF6 and IRE-1 as a result of its higher affinity with unfolded proteins, and then, the dissociated PERK, ATF6 and IRE-1 activate downstream c-JUN ammonia acid terminal kinase (c-JNK), CCAAT enhancer binding protein homologous protein (CHOP), cysteine aspartic proteinase -12 (caspase12) apoptotic signaling pathways, leading to cell apoptosis [7]. In JNK pathway, activated IRE-1 combines with tumor necrosis fector receptor associated factor 2 (TRAF2) through its intracytoplasmic binding domain to form IRE-1-TRAF2 complex, and then activates downstream apoptosis signal regulating kinase 1 (ASK1), and forms IRE-1-TRAF2-ASK1 complex with ASK1, which makes JNK phosphorylated and activated, subsequently, activated JNK inhibits the anti-apoptotic activity of BcL-2 and enhances the apoptotic function of BIM, also participate in the regulation of caspase-12, phosphorylate CHOP, and increase the apoptotic effect of each other by regulating CHOP activity [7-8]. Activated JNK can also activate activator protein-1 (AP-1) and increase the expression of pro-inflammatory factor genes. IRE1 $\alpha$-TRAF2 complex can also phosphorylate and degrade $\mathrm{IkB}$, release NF-kB into nucleus, activate the transcription of pro-inflammatory factor genes [9-10], lead to the increase of pro-inflammatory factors (TNF-a, IL-6, etc.) and increase the vulnerability of atherosclerotic plaques [11]. A large expression of endoplasmic reticulum chaperone was found in the samples obtained by percutaneous transluminal plaque rotated resection, and ERS apoptotic pathway was activated in unstable plaques [12].

Diabetes mellitus results in impairment of normal pathways of glucose metabolism, overactivation of four bypass pathways, including polyol pathway, protein kinase $\mathrm{C}$ (PKC) pathway, advanced glycation end products (AGEs) pathway and hexosamine pathway, which enhances the activity of NADPH oxidase and produces excessive reactive oxygen species (ROS) clusters in cells [13]. The accumulation of ROS affects the homeostasis of endoplasmic reticulum, which hinders the folding of protein spatial structure and induces the accumulation of unfolded proteins to induce ERS [14]. In addition, insulin resistance impairs the function of lipoprotein lipase, decreases the clearance rate of VLDL, and hyperinsulinemia promotes the synthesis of VLDL in the liver, presenting increased TG and LDL, decreased HDL and disordered lipid metabolism. Enhanced oxidative stress, which oxidizes LDL to ox-LDL, can not be recognized by LDL receptors for normal degradation, and has a high affinity with scavenger receptors of macrophages. This process has no negative feedback mechanism, leading to a large accumulation of cholesterol esters in macrophages, triggering ERS, which further exacerbating inflammatory response and leading to a vicious cycle [15]. Therefore, endoplasmic reticulum stress-coupled inflammation plays an important role in the occurrence and development of diabetic atherosclerosis. It has been found that insulin resistance can make macrophages more sensitive to endoplasmic reticulum stress-mediated apoptosis, induce inflammation, macrophage and smooth muscle cell apoptosis, and promote atherosclerosis occurrence and development [16-17]. Antioxidant enzyme system is an important barrier for the body's antioxidant injury, which mainly includes GSH-Px, SOD. GSH-Px is an important peroxidase widely existing in organisms, which is a detoxification enzyme, with the ability to catalyze the conversion of reduced glutathione (GSH) to oxidized glutathione (GSSG), reduce toxic peroxides to non-toxic hydroxyl compounds, promote $\mathrm{H}_{2} \mathrm{O}_{2}$ decomposition, remove hydrogen peroxide and other organic peroxides in organisms. SOD is a natural antioxidant enzyme of superoxide anion playing an important role in antioxidant damage. The synergistic effect of GSH-Px and SOD can prevent diabetes from producing excessive ROS.

According to TCM theory, deficiency of both Qi and Yin, dryness-heat accumulation are the basic pathogenesis of diabetic AS. Deficiency of Qi leads to blood stasis, Yin deficiency aggravates internal heat, heat and blood stasis can transform into toxin, phlegm and blood stasis can pile up over time (atherosclerotic plaque) [18]. At the same time, because inflammation is the main cause of the occurrence, development and plaque instability of AS, and the inflammation which causes infiltration of inflammatory cells and release of inflammatory mediators, similar to the phenomenon of heat toxicity, it suggest that antipyretic and detoxicating drugs can play a potential role in stabilizing plaques through anti-inflammation. Therefore, "supplementing Qi and nourishing Yin, activating blood circulation and detoxicating, softening hardness and eliminating accumulation" is an important therapy for diabetes AS."HJJF"(consists of Ginseng, Astragalus membranaceus, Ophiopogon japonicus, Cornus officinalis, Rehmannia glutinosa, Rhubarb, turtle shell, peach kernel, danpi, Coptis chinensis, Salvia miltiorrhiza, yam, Schisandra chinensis), in which, ophiopogon root, ginseng, schisandra are Jin Yuan Dynasty doctor Li Gao «Endogenous and Exogenous differentiation theory» Shengmai Powder ingredients, possessing nourishing Yin and tonifying Qi; cornus, rehmannia and yam are Ming Dynasty doctor Zhang Jingyue prescriptions "Zuo Gui pill" ingredients for nourishing kidney, treating true Yin deficiency; turtle shell, rhubarb, cortex moutan and peach kernel are Eastern Han Dynasty medical scientist Zhang Zhongjing's prescriptions 
"Turtle shell pills" ingredients for treaing "Accumulation"; With salvia miltiorrhiza activating blood and dispersing stasis, Huanglian Qingre clearing heat and damp-drying, and Astragalus supplementing qi, strengthening and detoxifying. It is an innovation in the treatment of diabetic AS to seek treatment ideas from TCM "nourishing Yin, Supplementing Qi, activating blood circulation, detoxifying, softening plaques and dissipating accumulation". Clinical studies have found that HJJF can inhibit insulin resistance, regulate anti-inflammatory/pro-inflammatory balance, check renin-angiotensin-aldosterone system (RAAS) in diabetic ACS patients, effectively curb inflammation in diabetic ACS patients with revascularization or non-revascularization, play a multi-link, multi-channel, multi-target role in overall regulation, improve. clinical effect and ameliorate cardiac function $[5,19]$.

This study showed that, After successful diabetes modeling, GSH-Px was activated to a certain extent and compensatory increased, but still insufficient to resist oxidative stress induced by high glucose. After drug intervention, FBG and SOD and GSH-Px were significantly decreased, lipid metabolism disorders were corrected (TC, TG, LDL-C contents were decreased, HDL-C were increased), levels of proinflammatory factors (TNF-a, IL-6) were lowered, and of ER apoptotic signaling molecules GRP78 and JNK transcription levels were down-regulated. HJJF treatment group was better than western medicine group, and the effect was more significant with increasing dose.

\section{Conclusion}

ERS is a common pathway of multiple stressors and a bridge between metabolic abnormalities and inflammation. The pathological states of diabetes mellitus, such as high sugar, high fat, oxidative stress and inflammation, are all related to ERS, which connects metabolic abnormalities and the development of diabetes mellitus, and causes diabetic atherosclerosis, therefor ERS will become a new target for the treatment of diabetes. The therapeutic effect of HJJF with the functions of nourishing Yin, invigorating Qi, activating blood circulation and detoxifying, lies in correcting insulin resistance, correcting lipid metabolism disorder, improving anti-oxidative stress ability, alleviating inflammatory reaction, inhibiting the coupling reaction of "oxidative stressendoplasmic reticulum stress-inflammatory response-cell apoptosis", and alleviating inflammation reaction of atherosclerotic plaque in diabetes mellitus, which embodies the prevention and treatment characteristics of integrated intervention, dynamic adjustment and multi-target therapy of traditional Chinese medicine.

\section{Acknowledgements}

This research is supported by National Natural Science Foundation of China (81460698); Guangxi emphasis research and development program (2017AB45042); Guangxi Natural Science Foundation (2015GXNSFAA139221).

\section{References}

[1] Ozcan U, Cao Q, Yilmaz E, et al. Endoplasmic reticulum stress links obesity, insulin action, and type 2 Diabetes [J]. Science, 2004, 306 (5695): 457-461.

[2] Wang M, Kaufman RJ. Protein misfolding in the endoplasmic reticulum as a conduit to human disease Nature, 2016, 529 (7586): 326-35.

[3] Sanson M, Auge N, Vindis C, et al. Oxidized low-density lipoproteins trigger endoplasmic reticulum stress in vascular cells: prevention by oxygen-regulated protein 150 expression. Cire Res, 2009, 104: 328-336.

[4] FU Xianzhao, XU Jing, HUANG Wenhua, et al. Strategy of Stasis-resolving and Detoxification on Diabetic Coronary Heart Disease [J]. JE TCM, 2014, 23 (11): 2024-2027.

[5] FU Xian-zhao, XU Jing, LI Chun-yan, et al. Effects of Huoxue Jiedu Jiangtang formulation on the balance between pro- and anti-inflammatory cytokines in non-revascularization patients with diabetic acute coronary syndrome [J]. Chin JTCM WM Crit Care, 2016, 23 (1): 6-10.

[6] Doultsinos D, Avril T, Lhomond S, et al. Control of the Unfolded Protein Response in Health and Disease. SLAS Discov, 2017, 22 (7): 787-800.

[7] GUAN LiYing, XU CaiMin, PAN Hua Zhen. Endoplasmic Reticulam Stress-induced Apoptosis. Prigress in Biochemistry and Biophysics, 2007, 11: 1136-1141.

[8] Hong F, Liu B, Wu BX, et al. CNPY2 is a key initiator of the PERK-CHOP pathway of the unfolded protein response. Nat Struct Mol Biol, 2017, 24 (10): 834-839.

[9] Zhang K, Kaufman R J. From endoplasmic-retieulum stress to the inflammatory response. Nature, 2008, 454: 455-462

[10] Lin JH, Walter P, Yen TS. Endoplasmic retieulum stress in disease pathogenesis. Annu Rev Pathol, 2008, 3: 399-425.

[11] Tabas I, Seimon T, Timmins J, et al. Macrophage apoptosis in advanced atherosclerosis. Ann N Y Acad Sci. 2009, 1173 (Suppl 1): E40-45.

[12] Lim W, Timmins J, Seimon T, et al. Signal transducer and activator of transcription-1 is critical for apoptosis in macrophages subjected to endoplasmic reticulum stress in vitro and in advanced atherosclerotic lesions in vivo [J]. Circulation, 2008. 117 (7): 940-951.

[13] Lindholm D, Korhonen L, Eriksson O, et al. Recent Insights into the Role of Unfolded Protein Response in ER Stress in Health and Disease. Front Cell Dev Biol, 2017, 10: 5-48.

[14] Zhang K, Kaufman RJ. From endoplasmic-reticulum stress to the inflammatory response [J]. Nature, 2008, 454: 455-462.

[15] Luchetti F, Crinelli R, Cesarini E, et al. Endothelial cells, endoplasmic reticulum stress and oxysterols. Redox Biol, 2017, 13: 581-587.

[16] Dickhou JG, Colgan SM, Lhotak S, et al. Increased endoplasmic reticulum stress in atherosclerotic plaques associated with acute coronary syndrome: A balancing act between plaque stability and rupture [J]. Circulation, 2007, 116 (11): 1226-1233. 
124 Fu Xianzhao et al: : Effect of Huoxue Jiedu Jiangtang Formulation on Endoplasmic Reticulum Stress in Diabetic Atherosclerosis

[17] Schmitz ML, Shaban MS, Albert BV, et al. The Crosstalk of Endoplasmic Reticulum (ER) Stress Pathways with NF-кB: Complex Mechanisms Relevant for Cancer, Inflammation and Infection [J]. Biomedicines, 2018, 6, 58; doi: 10.3390.

[18] Fu Xianzhao, Huang Zhenfeng, Huang Wenhua, et al. Review and prospect of using traditional Chinese medicine drugs "supplem enting Qi and nourishin $\mathrm{g}$ Yin, activating blood circulation and detoxifying" for prevention and treatment of diabetes mellitus complicated with acute coronary syndrome

[19] FU Xian-zhao, XU Jing, LI Chun-yan, et al. Effect of Huoxue Jiedu Jiangtang formulation on heart remodeling in diabetic patients with acute coronary syndrome after PCI [J]. Chin J TCM WM Crit Care, 2016, 23 (2): 117-121. 